\title{
Initial Results from the Experimental and Computational Study of Microbubble Generation
}

\author{
Alessio Basso $^{1}$, F. A. Hamad ${ }^{1}$, Poobalan Ganesan ${ }^{2}$ \\ ${ }^{1}$ School of Science Engineering \& Design, Teesside University \\ Southfield Road, Middlesbrough, TS1 3BA, UK \\ a.basso@tees.ac.uk; f.hamad@tees.ac.uk \\ ${ }^{2}$ Department of Mechanical Engineering, Faculty of Engineering, University of Malaya \\ Kuala Lumpur, Malaysia \\ poo_ganesan@um.edu.my
}

\begin{abstract}
A novel design of microbubble aeration is proposed to increase dissolved oxygen (DO) levels in water, by reducing the mean bubble diameter distribution down to the $20-50 \mu \mathrm{m}$ range. Microbubble generators (MBGs) find application in aquaculture farms, where water oxygenation is crucial for sea lives and for disinfection through free radicals. A Venturi MBG is taken as baseline design for comparison with previous literature, to understand the pressure-recovery mechanism responsible for bubble breakup. Based on Venturi performance, a helicoidal body - at three different pitch angles - is added ahead of the throat to create sufficient swirling motion, with the aim of intensifying turbulent pressure fluctuations. Results from a validated Computational Fluid Dynamics (CFD) setup and flow visualisation are compared to assess the accuracy of present experimental results, in terms of MBG performance at different volumetric qualities. Backlight imaging and binary processing are implemented to estimate microbubble distributions. Probability distribution and mean diameter plots show an initial disagreement of experimental data from the literature and present predictions. This is presumably attributed to a possible improvement to be done, in terms of light illumination and the use of a microscopic lens, which can better spot small microbubbles. Furthermore, results from CFD show a reduction in the mean diameter when using a helicoid pitch angle of 20 degrees. Of particular interest is a mean diameter near $25 \mu \mathrm{m}$ at very low volumetric quality. By contrast, the configuration at pitch angle of 30 degrees gives a distribution with mean diameter near $70 \mu \mathrm{m}$, for same volumetric quality. Although this paper only focuses on the bubble diameter aspect, DO measurements coupled with the analysis of microbubble distributions will reinforce authors assumption that small microbubbles represent the optimal condition to maximise water aeration and to enhance treatment of organic particles.
\end{abstract}

Keywords: Microbubble aeration, turbulent fluctuations, swirling flows, bubble breakup, CFD modelling, image analysis.

\section{Introduction}

Microbubble aeration is a common industrial process for waste-water disinfection - helpful due to the high levels of free radical produced [1, 2] - water recycling through dissolved air flotation (DAF) [3, 4, 5] and oil refinement [6], where oil droplet or particles attached to the bubble surface are brought to the water surface and separated from the continuous phase. Further applications regard bacteria digestion and fermentation [7], together with turbulent skin friction reduction [8, 9]. However, one of the most prominent usage of microbubbles is water oxygenation at sea living farms, e.g. shrimp aquaculture nurseries $[10,11]$. The direct effect of the increase of dissolved oxygen in animals is the expedition in their metabolism, hence, their rate of growth. As a result, the production of seafood is accelerated, together with a more efficient water treatment of bio-floc particles, such as faeces. In other words, a reduced usage of clean water would result in a smaller impact on environment and natural ecosystems.

Based on their diameter (D), bubbles have been classified in three different categories [1]: a) macrobubbles (D > 100 $\mu \mathrm{m}), \mathrm{b})$ microbubbles $(10 \mu \mathrm{m}<\mathrm{D}<100 \mu \mathrm{m})$, and c) nanobubbles $(\mathrm{D}<0.2 \mu \mathrm{m})$. The size of bubbles is of importance in relation with the amount of dissolved oxygen released in water. Indeed, macrobubbles are subject to a high rising speed (5.0 $-6.0 \mathrm{~m} / \mathrm{min}$ if $\mathrm{D}=1 \mathrm{~mm}[2,1])$, whereas microbubbles of diameter $\mathrm{D}=10 \mu \mathrm{m}$ can rise approximately at $3 \mathrm{~mm} / \mathrm{min}[12]$ and nanobubbles of diameter $\mathrm{D}=3.5 \mathrm{~nm}$ can indefinitely persist in water. Therefore, due to the high rising speed, macrobubbles do not have enough time to diffuse oxygen (DO) in water. By contrast, nanobubbles have a large internal pressure which, balanced by the water surface tension (inversely proportional to the curvature radius of the bubble) and 
pressure (equilibrium represented by the well-known Young-Laplace Equation [1]), prevents them to release dissolved oxygen. In fact, nanobubbles can persist for months before collapsing in water [13, 14]. For the reasons explained above and based on findings from previous works $[15,16]$, we have focussed our attention on small microbubbles which can effectively maximise the amount of dissolved oxygen released in a water aeration tank.

Thus far, different designs of microbubble generator (MBG) have been developed over the past few decades to reduce the mean diameter of the bubble distribution. Some made use of rotating perforated plates [16] where high-speed stirring could produce distributions with mean of the order of $20 \mu \mathrm{m}$. Other MBGs involved a tangential injection of air [17], perpendicular to the water flow direction in order to enhance bubble breakup through swirling flow rotation. Finally, some prototypes consist of a tube with an inner orifice [18] or a sphere [15] to produce a decrease in the internal static pressure to produce air suction from a near porous wall, fundamental for production of microbubbles. The present research focus converged on Venturi MBGs as they have the simplest and most energy-efficient design [19, 20]. A Venturi tube consists of a convergent section followed by a divergent section, where the compressible air-water mixture is injected and accelerated up to supersonic Mach speed $[8,9]$. In the above condition, bubbles coalesce in the convergent portion of the diffuser while abrupt normal shocks appear along the main axis of the generator behind the throat section (phenomena known as pressure recovery effect). This mechanism induces intense turbulent pressure fluctuations leading to bubble breakup, as opposing to the bubble internal pressure and surface tension. An additional effect in Venturi MBGs is the saturated-vapour pressure in the divergent section, which can result in cavitation [21].

In this work, we intend to improve the performance of current Venturi MBGs, through an innovative design, which can produce finer distributions with mean diameter down to the optimal range of 20 to $50 \mu \mathrm{m}$. With the above concept in mind, we have produced a special-machined aeration device, where a helicoidal geometry at three different pitch angles is added to induce swirling motion upstream of the tube throat. In this way, we aim at reducing microbubble coalescing at the throat and diminish the mean diameter at the outlet. Venturi baseline tube has been selected as a starting point for this research due to its low-energy requirement. In other words, we aim at saving more energy, while producing smaller microbubbles. Results from CFD analysis using the Rosin-Rammler distribution method and Bubble Breakup model in ANSYS Fluent are compared with flow visualisation data, where video frames are binary-processed to extract the mean diameters. Details are provided in the following sections.

\section{Materials and Methods}

In this section, the CFD setup and the experimental methodology for image analysis are explained in detail. Furthermore, the modified Venturi microbubble generators are presented. The numerical simulation has been validated by comparison with experimental data from the previous literature on the Venturi baseline design. All the modified Venturi prototypes have been assessed by using the same computational model.

The experimental setup includes the use of diffused light source to locally illuminate the water tank and to visualise microbubbles. A high-resolution camera (SONY DSC-RX10) has been used to record movies whose frames are then extracted and binary-processed. A series of morphological operations, including median filtering, morphological thinning and thickening, area opening, and skeletonisation are performed to remove any possible sources of error which could potentially lead to misleading results. For this reason, the cross correlation coefficient is calculated between the current and original images. Image parameters corresponding to each morphological operation are adjusted to obtain the optimal two-dimensional correlation coefficient. Bubble diameter distributions are determined by using the Hough transform method, as explained in the sections below.

\subsection{Microbubble generators and Experimental Setup}

Baseline and modified Venturi MBGs have been designed at Teesside University and manufactured by Paragon Rapid Technologies, based on drawings illustrated in [8] and [9]. A sample of the modified microbubble generator is shown in Figure 1, where a helicoidal material extrusion is added to the baseline Venturi tube, in front of the throat section. Designs described above are intended to increment the bubble breakup rate at the nozzle exit, in order to produce a fine uniform distribution of microbubbles smaller than $50 \mu \mathrm{m}$. Three different pitch angles are taken in consideration, namely $\beta=10^{\circ}, 20^{\circ}, 30^{\circ}$ alongside the baseline Venturi MBG. 


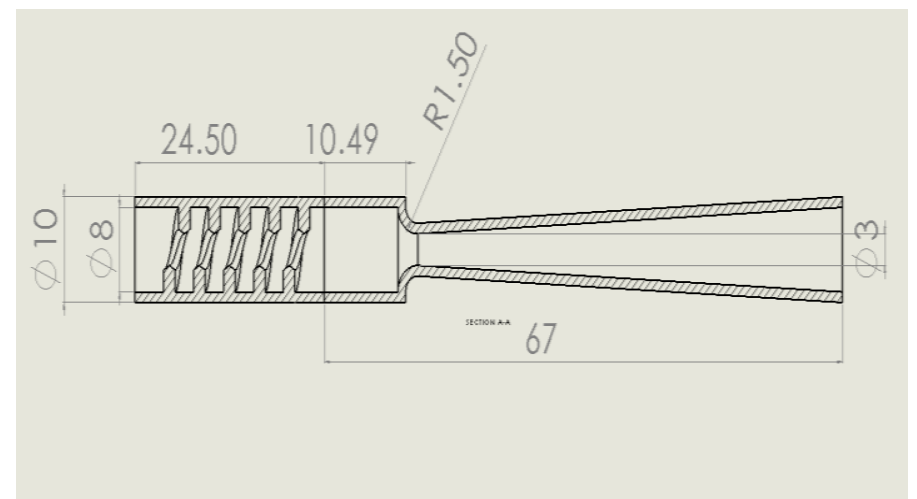

(a)

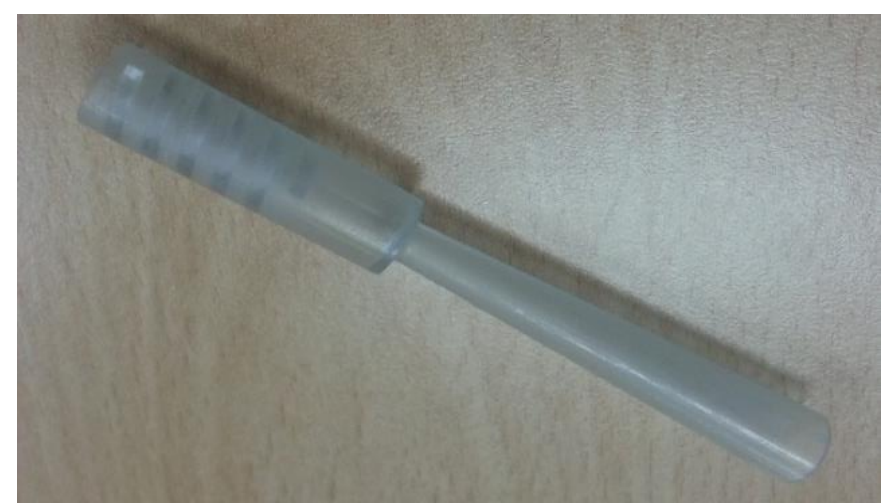

(b)

Fig. 1: Modified Venturi MBG, pitch angle $\beta=10^{\circ}$. Technical drawing (a) and manufactured model (b).

The experimental facility consists of a $380 \times 275 \times 440 \mathrm{~mm}$ water tank connected to a $0.665 \mathrm{~kW}$ pump producing a maximum flow rate of $10.0 \mathrm{l} / \mathrm{min}$ when no air is injected in the system. The microbubble generator is connected to one of the tank walls, and its inlet present a T-junction where compressed air and water mix together. A 20W halogen lamp is located on the opposite side with respect to the water tank, to illuminate the field of view of a SONY DSC-RX10 camera. Videos are recorded for each measurement at 25 frames/s to ensure that the same bubble is not processed more than once. In order to improve image quality, the water tank is obscured while diffused light is allowed through a $1 \mathrm{~mm}$ strip. In this way, microbubbles can be better focused whereas quantities such as working distance, focal length and depth of field are known and fixed. The experimental facility is shown in Fig. 2 below. Tests have been performed by fixing the air flow rate at 0.5 $1 / \mathrm{min}$ and variable water flow rates $(1.5-7.5 \mathrm{l} / \mathrm{min})$. Time interval between each test is large enough to allow all microbubbles to disappear from the field of view. Additionally, microbubbles are recorded after a certain time the MBG is off to permit flow settling.

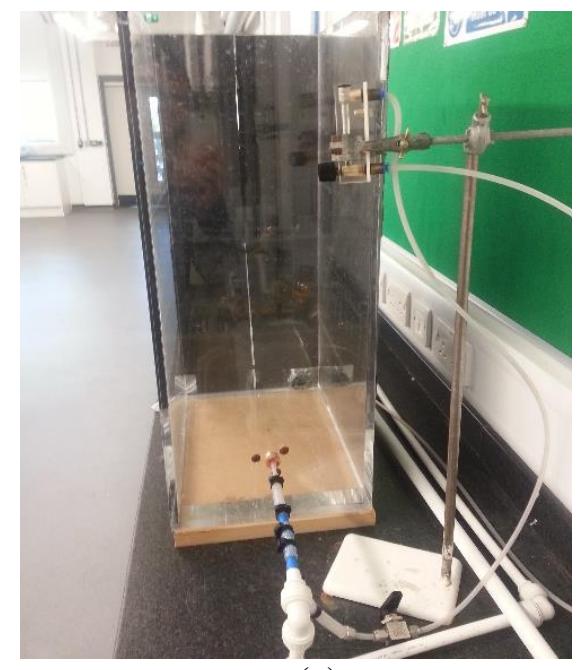

(a)

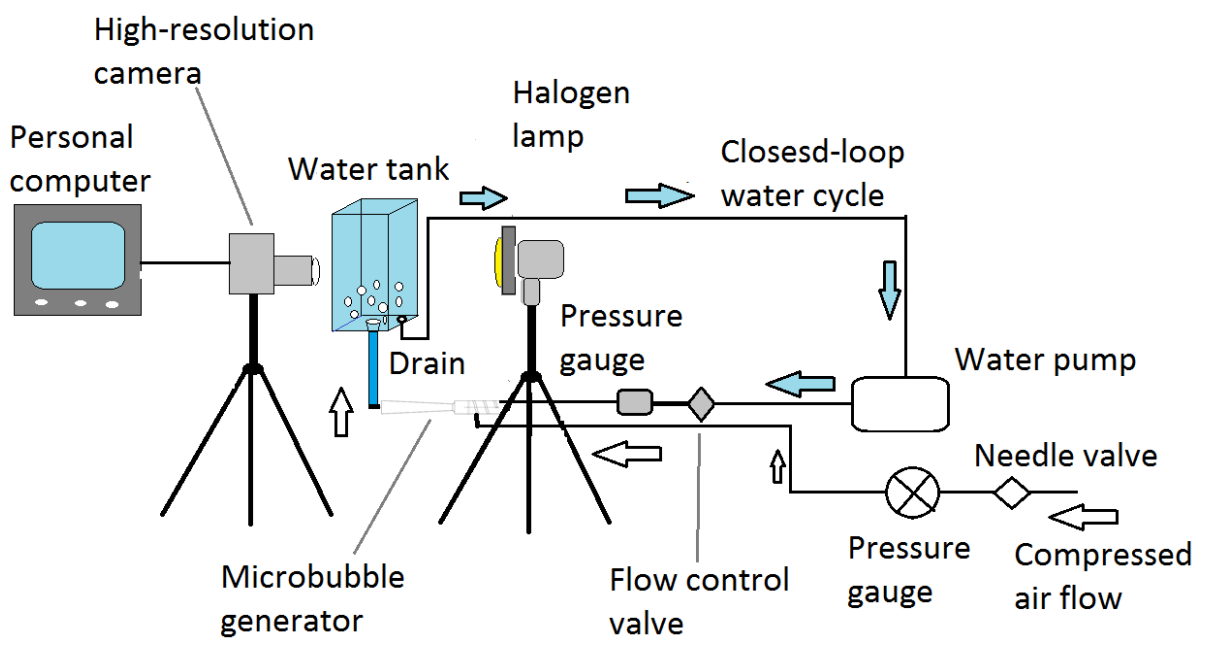

(b)

Fig. 2: Test rig at Teesside University (a) and setup schematic (b).

\subsection{CFD Setup and Numerical Procedure}

The numerical model has been setup in ANSYS Fluent v18.1. The air-water mixture has been modelled as a continuous phase of water with injection of dispersed gas particles to simulate microbubbles. Therefore, the Lagrangian Discrete Phase Model (DPM) has been used to predict the instantaneous particle trajectory, based on the dynamic balance of forces acting on the particle surface, namely inertia, viscous drag and gravitational field, as shown in Eqs. (1) - (2) below. 


$$
\begin{gathered}
\frac{d u_{p}}{d t}=F_{D}\left(u-u_{p}\right)+\frac{g_{x}\left(\rho_{p}-\rho\right)}{\rho_{p}} \\
F_{D}=\frac{18 \mu}{\rho_{p} d_{p}^{2}} \frac{C_{D} R e}{24}
\end{gathered}
$$

The first term from left on the right side of Eq. (1) is the drag force per unit mass of particle, $F_{D}$ the drag force on particle, whereas $\left(u-u_{p}\right)$ is the relative velocity of the dispersed particles in the continuous phase. In Eq. (2), $\rho$ is the continuous phase velocity, $C_{D}$ is the drag coefficient when the particle is an ideal sphere, $\mu$ is the viscosity of continuous phase, $\rho_{p}$ and $d_{p}$ are the density and diameter of dispersed particles, respectively. The Reynolds number $(R e)$ of dispersed particles is defined in Eq. (3):

$$
R e=\frac{\rho d_{p}\left|u_{p}-u\right|}{\mu}
$$

The Rosin-Rammler Method has been selected to model the microbubble distribution at the inlet surface used to inject air. The algorithm provides an exponential distribution of $d_{p}$ with the mass fraction of larger particles, as shown in Eq. (4), where $n$ is the size distribution parameter and $\bar{d}$ a constant:

$$
Y_{d}=e^{-(d / \bar{d})^{n}}
$$

The continuous phase of water is modelled as incompressible fluid through the Reynolds-Averaged Navier-Stokes set (RANS), including continuity and momentum equations, namely Eqs. (5) - (6) respectively, having assumed negligible thermal effects:

$$
\begin{gathered}
\frac{\partial\left(\rho u_{i}\right)}{\partial x_{i}}=S_{m} \\
\frac{\partial\left(\rho u_{i} u_{j}\right)}{\partial x_{j}}=-\frac{\partial p}{\partial x_{i}}+\frac{\partial}{\partial x_{j}}\left[\mu\left(\frac{\partial u_{i}}{\partial x_{j}}+\frac{\partial u_{j}}{\partial x_{i}}\right)\right]+\frac{\partial\left(-\rho \overline{u_{\imath}^{\prime} u_{j}^{\prime}}\right)}{\partial x_{j}}+\rho g+F
\end{gathered}
$$

Where $u_{i}$ and $u_{j}$ are the average in $\mathrm{x}$ and y direction, respectively, $S_{m}$ is the mass source term added to the system through dispersion of the second phase, $\mathrm{p}$ is the pressure, $F$ is the additional body force added through interaction with particles, $u i^{\prime}$ and $u j^{\prime}$ are the turbulent fluctuation in $\mathrm{i}$ or $\mathrm{j}=1$ correspond to the $\mathrm{x}$-direction, $\mathrm{i}$ or $\mathrm{j}=2$ the $\mathrm{y}$-direction and $\mathrm{i}$ or $\mathrm{j}=3$ the z-direction.

Furthermore, the Standard $k-\varepsilon$ model is used to provide an estimation of the turbulent viscosity $\mu_{t}$, modelled as being proportional to the square of the kinetic energy $(k)$ and inversely proportional to the dissipation rate $(\varepsilon)$ :

$$
\begin{gathered}
\mu_{e f f}=\mu+\mu_{t} \\
\mu_{t}=C_{\mu} \rho \frac{k^{2}}{\varepsilon}
\end{gathered}
$$

Where $C_{\mu}$ is a constant (0.09). Finally, the Wave Breakup model [22] is used to simulate the breakup of bubbles as a result of the pressure fluctuations occurring from swirling motion and pressure recovery effects.

Simulation has been run in the form of unsteady flow, where a reasonable time step has been set to capture the instantaneous particle trajectory in order to satisfy the CFL criterion for an explicit-solver formulation - i.e. maximum 
Courant number equal to 1 . In order to prevent numerical diffusion and solution instability in the final result, and since tetrahedral elements have been used in the current CFD model - which also precludes high-level of flow mixing and separation along the main domain direction - a second-order Upwind spatial scheme has been selected for these numerical simulations.

The mesh grid has been produced and refined in ANSYS Meshing v18.1, and validated through mesh-independence study. A further convergence criterion has been set in the maximum net mass-flow unbalance to be equal to $1 \%$ between inlets and outlet sections, in order to guarantee mass continuity condition. Residuals convergence was set to $1.0 \mathrm{e}^{-05}$ to ensure a high-accuracy solution.

The grid independence study has been discussed in details in our previous paper (Basso et al. [23]) where the same CFD model is presented and fully-validated with previous experimental and numerical data from the literature. A valid mesh grid size had been estimated by varying the total number of elements in the grid domain and by keeping the same inflation layer ratio near the wall. In other words, when moving away from the wall, the current cell size at given distance from the wall is a linear function of the wall-attached cell, i.e. the first layer cell adjacent to the wall. Therefore, mesh grid independence had been determined by varying the size of the first wall-attached fluid cell. The average diameter of the microbubble distribution at the domain outlet is then taken as a reference parameter to evaluate grid independence.

\subsection{Image Analysis}

An image analysis procedure has been developed to extract bubble diameter distributions from video frames recorded by the high-resolution camera. A code has been developed in MATLAB 2016a software for binary image processing and cross correlation. Binary processing is divided into three different stages and includes morphological operations such as area opening, median filtering, thinning, thickening, image filling, and skeletonisation. In each operation, pixels are removed or added with the final aim of maximising the correlation coefficient between the current image with the original movie frame. Furthermore, median filtering is crucial for edge detection as it can remove image noise while preserving the contour of binary objects. Size of the cell for median filter is also selected on an image correlation basis.

Bubble diameter extraction is performed through the Hough Transform, where a circle parameters become known variables when a number of points falls within its perimeter [24]. For instance, a circle of radius $R$, centred in the point of coordinates $(a, b)$, is defined by the system in Eqs. (9) - (10), being $\vartheta$ an angle spanning from $0^{\circ}$ to $360^{\circ}$ :

$$
\begin{aligned}
& x=a+R \sin \theta \\
& y=b+R \sin \theta
\end{aligned}
$$

In other words, the Hough transform searches for all the circles identified through the parameter triplet $(a, b, R)$. Therefore, when the radius is known and has fixed value, perimeter searched is the locus of points $(x, y)$ falling on a circle of radius $R$ and centre in $(a, b)$. By contrast, whenever a range of radii is defined, the loci of points $(x, y)$ will fall on a conical surface defined by the maximum and minimum values of the radius. In the latter case three-dimensional matrix accumulation is applied for circle searching. In the current application, the algorithm described above has been set to search circles with radius ranging from $20 \mu \mathrm{m}$ to $350 \mu \mathrm{m}$. The strongest $95 \%$ of contours were detected. Results of the image analysis procedure are shown in Figure 3 below:

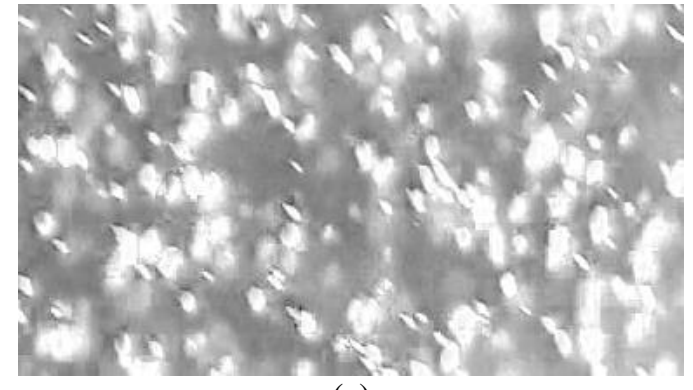

(a)

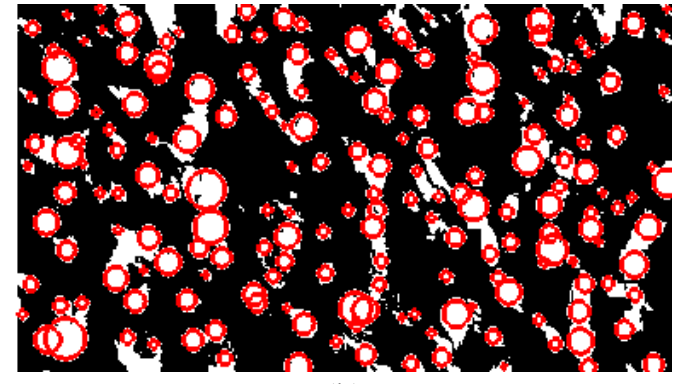

(b)

Fig. 3: Image Analysis: original frame (a) and final processed image with detected bubbles (b). 


\section{Results and Discussions}

The three different modified configurations have been compared, together with the baseline Venturi generator and the experimental results from Kawashima's work [9]. This was done in order to validate the current CFD model, as well as to assess the accuracy of results from our test rig. Note that experiments could not be carried out at exactly the same volumetric qualities as Kawashima's tests. This is due to a practical challenge in present test rig in controlling the air flow rate when the water pump was on.

Results in Fig. 4-a show a good agreement between the CFD model and measurements from Kawashima. This proves that present numerical model has been correctly validated. By contrast, the bubble distributions from present test rig and method produce smaller bubbles, in the range of $50-70 \mu \mathrm{m}$. This is encouraging, as it means that present experimental results might reflect a more accurate diameter distribution of microbubbles.

Similarly, we have compared CFD simulation and experimental results from the test rig to assess the three modified configurations, in Fig. 4-b, Fig. 4-c, 4-d namely at helicoid pitch angles $\beta=10^{\circ}, 20^{\circ}, 30^{\circ}$. It can be observed that, while experimental measurements show a diameter range of $50-70 \mu \mathrm{m}$ for all three volumetric qualities, numerical data suggest that: a) at $\beta=10^{\circ}$ the distribution shifts to the left, i.e. to smaller mean diameters, when increasing the volumetric quality; b) at $\beta=20^{\circ}$ both low and high volumetric qualities produce similar values of the mean diameter in the range of 20-40 $\mu \mathrm{m}$, hence, smaller that experimental results from present test rig; c) at $\beta=30^{\circ}$ again we obtain mean diameter distributions near $20 \mu \mathrm{m}$ at $\alpha=20 \%$. In other words, CFD simulation seems to suggest that an increase in the volumetric quality $\alpha$ produces a finer and more uniform distribution of microbubbles.

We explain the above discrepancy between numerical and experimental data with a necessity to improve the experimental method, especially the illumination approach. In fact, diffused light illuminates microbubbles which are away from the focal plane. As a result, the number and size of white circles in the corresponding binary image (Fig. 3) are affected, together with the final bubble distribution. Therefore, we believe it is worth, in a further development, to repeat tests with a coherent laser sheet light which, within a small thickness, can only illuminate bubbles inside the focal plane, or at least within a minimised distance. In this way, the error produced by out-of-focus bubble becomes negligible. An additional improvement has been identified in the use of a microscopic lens system to magnify the field of view, before the image frame is processed. In this way, even the smaller microbubbles in the distribution are considered in the image processing step.

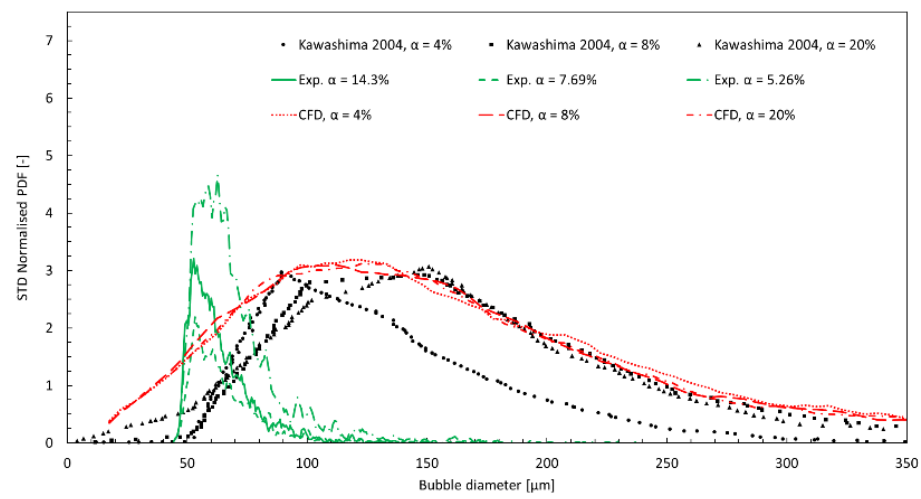

(a)

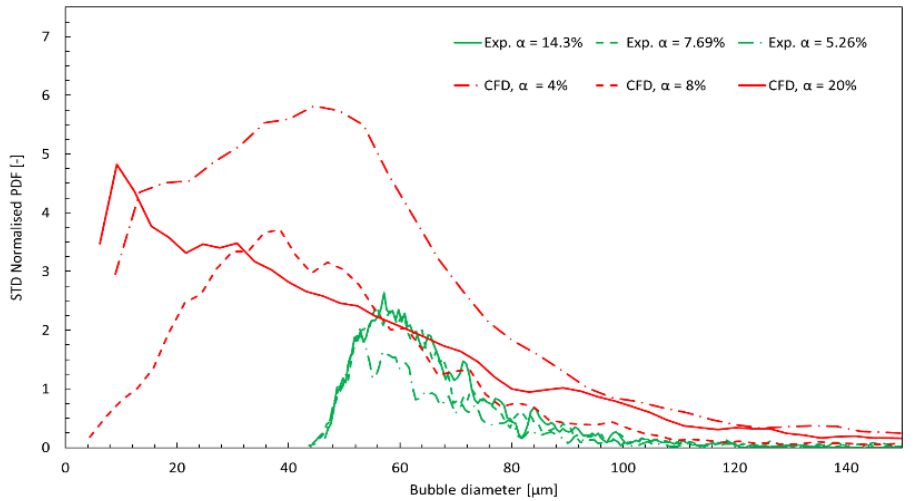

(b) 


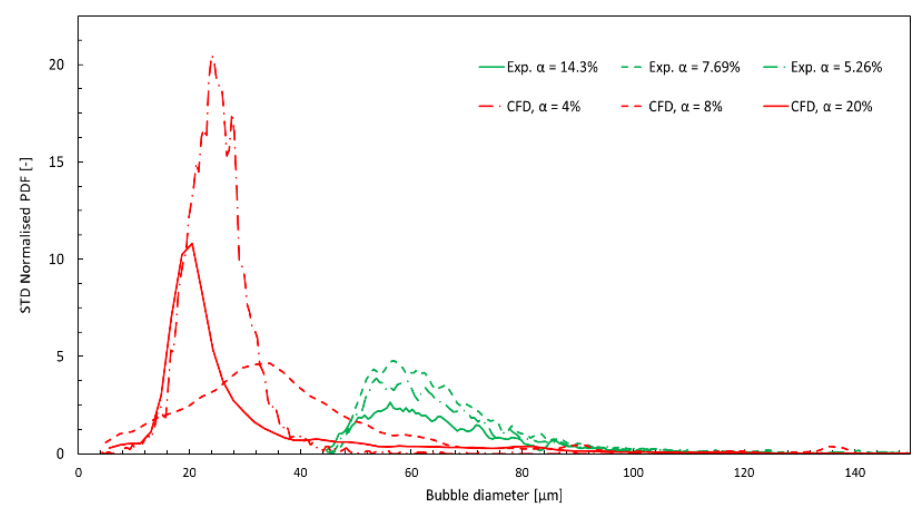

(c)

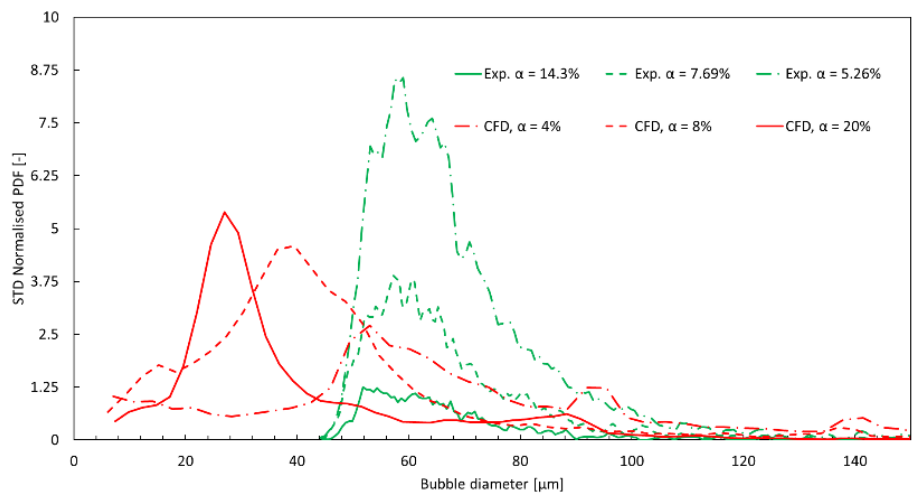

(d)

Fig. 4: Microbubble distributions for baseline (a) and modified Venturi generators - helicoid pitch angle $\beta=10^{\circ}$ (b), $20^{\circ}$ (c), $30^{\circ}$ (d).

Results above have further been cross-checked by plotting the actual values of the mean diameters for all configurations and volumetric qualities (Fig. 5).

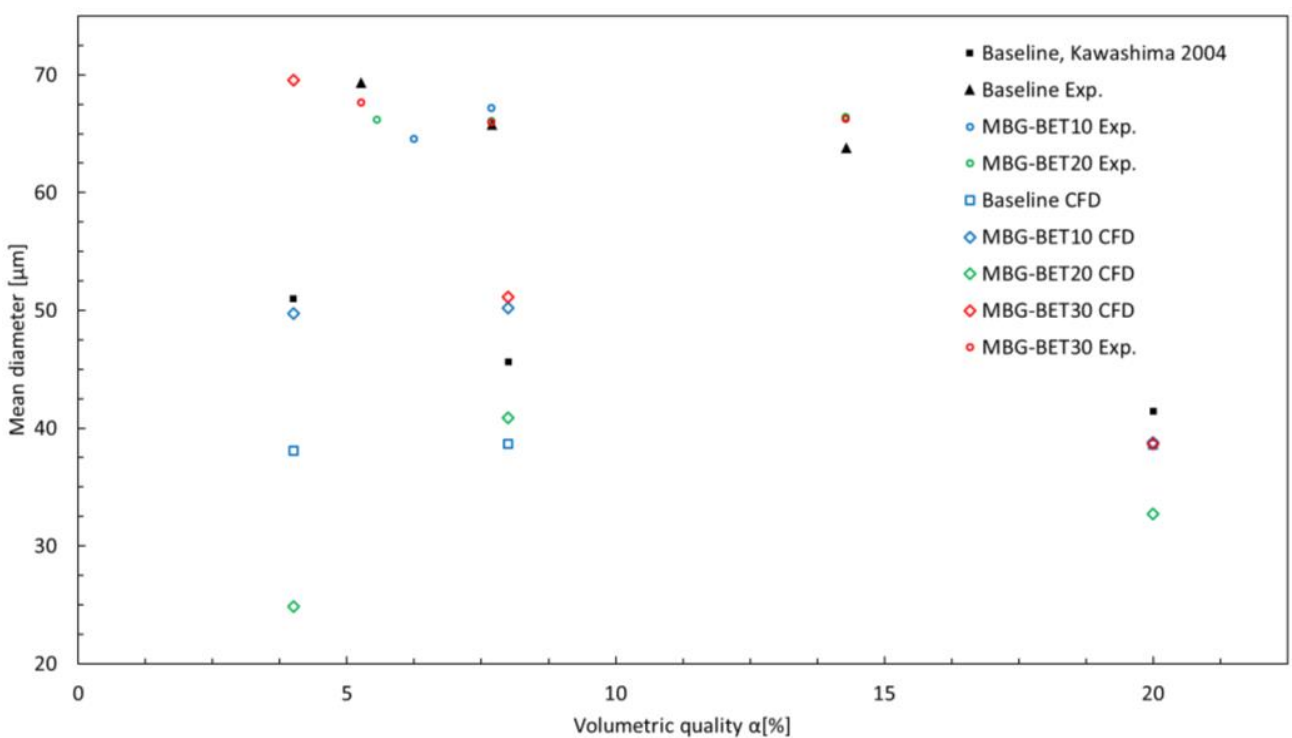

Fig. 5: Mean diameter versus volumetric quality for all measurements and numerical results.

From Fig. 5, the present experiment for the baseline case shows significant discrepancy with Kawashima's data. However, the numerical simulation suggests variation below $12 \%$ at volumetric quality $\alpha=20 \%$. Furthermore, as mentioned above, all results from the current work show mean diameter between 63.5 and $70 \mu \mathrm{m}$, while numerical data gives $25-53 \mu \mathrm{m}$. Particularly, while modified configurations at pitch angles equal to $10^{\circ}$ and $30^{\circ}$ perform similarly at volumetric qualities $\alpha$ equal to $7 \%$ and $20 \%$, the geometry with helicoidal pitch $\beta=20^{\circ}$ gives the best performance, especially at volumetric quality $\alpha=4 \%$, where mean diameter is approximately equal to $25 \mu \mathrm{m}$. By contrast, the worst performance is from the geometrical configuration at $\beta=30^{\circ}$ and $\alpha=4 \%$, where a mean diameter of approximately $70 \mu m$ is observed.

This work is based on our previous numerical model (Basso et al. [23]) which was validated with numerical and experimental results from other designs of microbubble generators in the literature. MBGs performance is estimated in terms of Probability Density Function (PDF) of the microbubble diameter distribution, especially in the computation of the mean diameter - in relation with the levels of dissolved oxygen released in water. The main scope of the work presented in this paper is to present a preliminary assessment of our experimental results and methodology, in view of the previous numerical data from our CFD model. For completion, some results are also already compared in Fig. 4A with Kawashima's ones (Kawashima et al. [9]). 
Experimental Uncertainty: The overall uncertainty is the result of error propagation from different sources: uncertainty from the sensor, the relative distance between the camera lens and focal plane, the calibration procedure (i.e. the conversion from pixel to $\mathrm{mm}$ and vice versa). Additionally, there is a so-called motion-blur uncertainty simply due to the fact that microbubbles are moving at certain speed in the field of view. The work by Robbe et al. [25] gives an idea of the complexity in estimating uncertainty in high-speed camera measurements. In the current preliminary work, we have not performed an uncertainty study, due to the complexity of the variables involved. However, the calibration process has been fully-validated with images of known size and resolution, while results were directly compared with other established data. Therefore, we would propose the uncertainty estimation as a further procedure for future developments of the methodology currently implemented in this study.

\section{Conclusions and future developments}

The present work aims at reducing the mean diameter of a microbubble distribution, in order to increase DO levels in water. Three geometrical configurations have been compared with a Venturi MBG, by adding a helicoidal element at three different pitch angles. An optimal geometry and flow condition has been identified in the pitch angle of $\beta=20^{\circ}$ and volumetric quality $\alpha=4 \%$, where a mean bubble diameter of the order of $25 \mu \mathrm{m}$ has been achieved. At high volumetric qualities $(\alpha=20 \%)$ the mean distribution was below $35 \mu \mathrm{m}$, which suggests an improvement from the baseline case.

A discrepancy is observed between experiments and CFD results which is attributed to the illumination method and the necessity of a microscopic lens to reduce the error due to out-of-focus bubbles. Results from this work are promising, especially for aquaculture farms, where it is crucial to accelerate the animal growth rate while reducing the environmental impact due to water effluents treatment.

\section{Acknowledgements}

The authors acknowledge EPSRC for funding this project under the grant agreement no. EP/P018211/1, in collaboration between Teesside University and University of Malaya.

\section{References}

[1] T. Marui, "An introduction to micro/nano bubbles and their applications" in The 14th World Multi-Conference on Systemics, Cybernetics and Informatics, vol. 1, 2010.

[2] A. Argawal, W. Jern and Y. Liu, "Principle and applications of microbubble and nanobubble technology for water treatment" in Chemosphere, vol. 84, pp. 1175-1180, 2011.

[3] S. Yoo and J. Hsieh, "Advanced water recycling through electrochemical treatment of effluent from dissolved air flotation unit of food processing industry” in Water Science Technology, vol. 61, pp. 181-190, 2010.

[4] J. Edzwald and J. Haarhoff, McGraw-Hill, 2012.

[5] J. Rubio, E. Carissimi and J. Rosa, "Flotation in water and wastewater treatment and reuse: recent trends in Brazil" in International Journal of Environment and Pollution, vol. 30, pp. 197-212, 2007.

[6] A. Moursy and S. Abo El-Ela, "Treatment of oil refinery waste using dissolved air flotation process" in Environ. Int., vol. 7, pp. 267-270, 1982.

[7] S. Himuro, T. Deguchi and R. Takamatsu, "Effects of Microbubbles on Bacteria" in Progress in Multiphase Flow Research, vol. 4, pp. 95-102, 2009.

[8] T. Kawamura, A. Fujiwara, T. Takahashi, H. Kato, Y. Matsumoto and Y. Kodama, "The effects of the bubble size on the bubble dispersion and skin friction reduction" in Proceedings of the 5th symposium on smart control of turbulence, Tokyo, 2004.

[9] H. Kawashima, A. Fujiwara, Y. Saitoh, K. Hishida and Y. Kodama, "Experimental study of frictional drag reduction by microbubbles: laser measurement and bubble generator" in Proceedings of the fifth symposium on smart control of turbulence, Tokyo, 2004.

[10] A. Chowdhuri, A. Talib and K. Yahya, "A review on marine shrimp aquaculture production trend in Malaysia and the world perspective," 2013. 
[11] H. Ohnari, "Fisheries experiments of cultivated shells using micro-bubbles technique" in Journal of the Heat Transfer Society of Japan, vol. 40, pp. 2-7, 2001.

[12] A. Endo, S. Srithongouthai, H. Nashiki, I. Teshiba, T. Iwasaki, D. Hama and H. Tsutsumi, "DO-increasing effects of a microscopic bubble generating system in a fish farm" in Marine Pollution Bulletin, vol. 57, pp. 78$85,2008$.

[13] M. Takahashi, T. Kawamura, Y. Yamamoto, H. Ohnari, S. Himuro and H. Shakutsui, "Effect of shrinking microbubble on gas hydrate formation" in The Journal of Physical Chemistry, vol. 107, no. 10, 2003.

[14] M. Takahashi, "Constriction and collapse of micro-bubbles" in Lecture Series of the Japanese society for Multiphase Flow, vol. 28, pp. 15-19, 2003.

[15] M. Sadatomi, A. Kawahara, K. Kano and A. Ohtomo, "Performance of a new micro-bubble generator with a spherical body in a flowing water tube" in Experimental Thermal and Fluid Science, vol. 29, pp. 615-623, 2005.

[16] Fujikawa and Shigeo, "The control of micro-air-bubble generation by a rotational porous plate" in International Journal of Multiphase Flow, vol. 29, no. 8, pp. 1221-1236, 2003.

[17] K. Tabei, S. Haruyama and S. Yamaguchi, "Study of micro bubble generation by a swirl jet" in Transactions of the Japanese Society for Mechanical Engineering, vol. 71, no. 703B, pp. 848-853, 2007.

[18] M. Sadatomi, A. Kawahara, H. Matsuura and S. Shikatani, "Micro-bubble generation rate and bubble dissolution rate into water by a simple multi-fluid mixer with orifice and porous tube" in Experimental Thermal and Fluid Science, vol. 41, pp. 23-30, 2012.

[19] M. Ishikawa, K. Irabu, I. Teruya and M. Nitta, "PIV measurement of a contraction flow using micro-bubble tracer" in The sixth symposium on measurement techniques for multi-phase flows, 2009.

[20] A. Gordiychuk, M. Svanera, S. Benini and P. Poesio, "Size distribution and Sauter mean diameter of micro bubbles for a Venturi type bubble generator" in Experimental Thermal and Fluid Science, vol. 70, pp. 51-60, 2016.

[21] A. Yoshida, O. Takahashi, Y. Ishii, Y. Sekimoto and Y. Kurata, "Water Purification Using the Adsorption Characteristics of Microbubbles" in Japanese Journal of Applied Physics, vol. 47, no. 8R, 2008.

[22] ANSYS Inc. ANSYS Fluent User manual [Online]. Available: http://www.ansys.com/Industries/Academic/Tools/Citations

[23] Basso A, Hamad FA, Ganesan P. Effects of the geometrical configuration of air-water mixer on the size and distribution of microbubbles in aeration systems. Asia-Pac J Chem Eng. 13 2259. 2018.

[24] H. Rhody and C. Carlson. (2005, October). Lecture 10: Hough Circle Transform [Online]. Available: https://www.cis.rit.edu/class/simg782/lectures/lecture_10/lec782_05_10.pdf

[25] C. Robbe, N. Nsiampa, A. Oukara and A. Papy1, "Quantification of the uncertainties of high-speed camera measurements", Int. J. Metrol. Qual. Eng. 5, 201, 2014. 